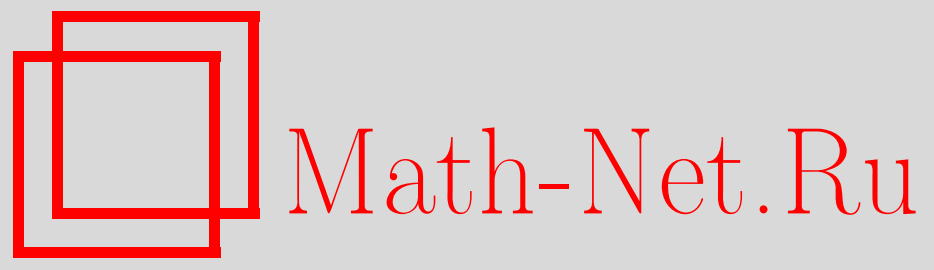

A. Philippe, D. Surgailis, M.-C. Viano, Time-Varying Fractionally Integrated Processes with Nonstationary Long Memory, Теория вероятн. и ее примен., 2007, том 52, выпуск 4, 768-792

DOI: https://doi.org/10.4213/tvp1533

Использование Общероссийского математического портала Math-Net.Ru подразумевает, что вы прочитали и согласны с пользовательским соглашением

http://www . mathnet.ru/rus/agreement

Параметры загрузки:

IP : 54.196 .121 .252

26 апреля 2023 г., 12:37:35

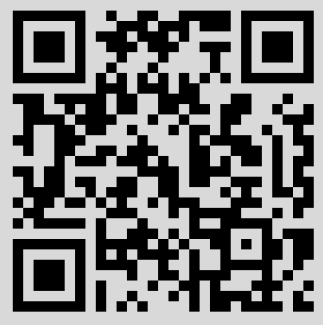




\title{
TIME-VARYING FRACTIONALLY INTEGRATED PROCESSES WITH NONSTATIONARY LONG MEMORY ${ }^{1)}$
}

\begin{abstract}
В работе вводятся два явных класса $A(\mathbf{d}), B(\mathbf{d})$ линейных фильтров, зависяших от времени и определяемых для любой вешественной последовательности $\mathbf{d}=\left(d_{t}, t \in \mathbf{Z}\right)$, таких, что для постоянной последовательности $d_{t} \equiv d$ операторы $A(\mathbf{d})=B(\mathbf{d})=(I-L)^{-d}$ coвпадают с обычным оператором дробного дифференцирования. Доказано, что эти операторы удовлетворяют соотношениям обратимости $B(-\mathbf{d}) A(\mathbf{d})=A(-\mathbf{d}) B(\mathbf{d})=I$. В работе исследуется асимптотическое поведение частных сумм фильтрованных процессов белого шума $Y_{t}=A(\mathbf{d}) G \varepsilon_{t}$ и $X_{t}=B(\mathbf{d}) G \varepsilon_{t}$ в случае, когда последовательность $\mathrm{d}$ имеет пределы $\lim _{t \rightarrow \pm \infty} d_{t}=d_{ \pm} \in\left(0, \frac{1}{2}\right)$ в бесконечности, а оператор $G$ образует фильтр с короткой памятью. Доказано, что пределом частных сумм является автомодельный гауссовский процесс, зависящий только от предельных значений $d_{ \pm}$и суммы коэффициентов оператора $G$. Кроме того, предельный процесс имеет либо асимптотически стационарные, либо асимптотически стремящиеся к нулю приращения и гладкие траектории.
\end{abstract}

Ключевые слова и фразы: нестационарная дальняя память, зависяшее от времени дробное интегрирование, частные суммы, автомодельные процессы, асимптотически стационарные приращения.

1. Introduction. Long memory, also called long-range dependence, is one of the most dynamically developing areas of probability and statistics; see, e.g., the recent volume [8]. From the theoretical probability point of view, the most interesting feature of long-range dependence concerns nonclassical limit behavior of the partial sums process. Recall that a stationary time series $\left(X_{t}, t \in \mathbf{Z}\right)$ is said to be long-range dependent if its partial sums

* Laboratoire de Mathématiques Jean-Leray, UMR CNRS 6629, Université de Nantes, F-44322 Nantes Cedex 3, France; e-mail: anne.philippe@math.univ-nantes.fr

** Institute of Mathematics and Informatics, Akademijos 4, LT-08663 Vilnius, Lithuania; e-mail: sdonatas@ktl.mii.It

*** Laboratoire Paul Painlevé UMR CNRS 8524, UFR de Mathématiques — Bat M2, Université de Lille 1, Villeneuve d'Ascq, 59655 Cedex, France; e-mail: marieclaude.viano@univ-lille1.fr

1) This research was supported by the bilateral France-Lithuania scientific project Gilibert and the Lithuanian State Science and Studies Foundation (grant № T-15/07). 
process, when suitably normalized, weakly converges to some random process with dependent increments, see, e.g., [5, p. 59], [7, p. 76-78]. Under weak additional assumptions, the limit process is self-similar [11].

Probably the most popular stationary long memory processes are the $\operatorname{FARIMA}(p, d, q)$ defined by the equations $\phi(L) X_{t}=\vartheta(L)(I-L)^{-d} \varepsilon_{t}$, where $\left(\varepsilon_{t}, t \in \mathbf{Z}\right)$ is a zero-mean white noise, $I$ is the identity, $L$ is the backward shift operator, $\phi(L), \vartheta(L)$ are polynomials in $L$ of degree $p, q$, respectively. Recall that the fractional differencing filter $(I-L)^{-d}\left(0<d<\frac{1}{2}\right)$ is defined by the binomial expansion

$$
(I-L)^{-d} x_{t}=\sum_{j=0}^{\infty} \psi_{j}(d) L^{j} x_{t}=\sum_{j=0}^{\infty} \psi_{j}(d) x_{t-j}
$$

where $\psi_{0}(d):=1$ and

$$
\psi_{j}(d):=\frac{d}{1} \frac{d+1}{2} \cdots \frac{d-1+j}{j}=\frac{\Gamma(d+j)}{j ! \Gamma(d)} \quad(j \geqslant 1) ;
$$

see, e.g., [3]. It is well known that, under suitable conditions on the polynomial function $\phi(\cdot)$ and $\left(\varepsilon_{t}, t \in \mathbf{Z}\right)$, the autocovariance function of the $\operatorname{FARIMA}(p, d, q)$ process $X_{t}$ decays as $t^{2 d-1}$, and the partial sums process $N^{-d-1 / 2} \sum_{t=1}^{[N \tau]} X_{t}$ converges in distribution to a fractional Brownian motion $(\mathrm{fBm}) W_{H}(\tau)$ with Hurst parameter $H=d+\frac{1}{2}$. The convergence of partial sums for general stationary second order linear processes was obtained in [6]. There it is proved that, in the case of i.i.d. noise, the limiting process is always a $\mathrm{fBm}$, a self-similar Gaussian process with dependent increments and nondifferentiable sample paths. In [12] it is proved that the above mentioned convergence result still holds under rather weak assumptions on the noise. Considerable attention in the literature was also given to limit theorems for partial sums processes of nonlinear instantaneous filters of stationary FARIMA and more general stationary linear processes with long memory; see, e.g., [18] for recent developments.

Until recently, long-range dependence has been studied almost exclusively in the stationarity setup. One of the reasons for this is the lack of appropriate models with nonstationary long memory.

In the present paper we provide a family of such processes presenting long memory and study the limiting behavior of their partial sums.

Starting from (1.1), we introduce the time-varying fractional differentiation operators

$$
A(\mathbf{d}) x_{t}=\sum_{j=0}^{\infty} a_{j}(t) x_{t-j}, \quad B(\mathbf{d}) x_{t}=\sum_{j=0}^{\infty} b_{j}(t) x_{t-j}
$$

where $\mathbf{d}=\left(d_{t}, t \in \mathbf{Z}\right)$ is a given function of $t \in \mathbf{Z}$, and where $a_{0}(t)=$ 


$$
\begin{aligned}
& b_{0}(t):=1, a_{1}(t)=b_{1}(t):=d_{t-1}, \\
& a_{j}(t):=\frac{d_{t-1}}{1} \frac{d_{t-2}+1}{2} \frac{d_{t-3}+2}{3} \ldots \frac{d_{t-j}+j-1}{j} \\
&=\prod_{k=1}^{j} \frac{k-1+d_{t-k}}{k}, \quad j \geqslant 2, \\
& b_{j}(t):=\frac{d_{t-1}}{1} \frac{d_{t-j}+1}{2} \frac{d_{t-j+1}+2}{3} \ldots \frac{d_{t-2}+j-1}{j} \\
&=\frac{d_{t-1}}{1} \prod_{k=2}^{j} \frac{k-1+d_{t-j+k-2}}{k}, \quad j \geqslant 2 .
\end{aligned}
$$

If $d_{t} \equiv d$ is a constant, then $A(\mathbf{d})=B(\mathbf{d})=(I-L)^{-d}$ is the usual fractional differentiation operator. Note that both $a_{j}(t)$ and $b_{j}(t)$ depend on $\left(d_{t-1}, \ldots, d_{t-j}\right)$ and that $b_{j}(t)$ is obtained from $a_{j}(t)$ by time reversing

$$
\left(d_{t-2}, d_{t-3}, \ldots, d_{t-j+1}, d_{t-j}\right) \longrightarrow\left(d_{t-j}, d_{t-j+1}, \ldots, d_{t-3}, d_{t-2}\right) .
$$

A remarkable property of these operators is that $B(-\mathrm{d})$ is the inverse of $A(\mathbf{d})$ and vice versa; in other words,

$$
B(-\mathbf{d}) A(\mathbf{d})=A(-\mathbf{d}) B(\mathbf{d})=I
$$

where $-\mathbf{d}:=\left(-d_{t}, t \in \mathbf{Z}\right)$. The invertibility property is very important in regard to applications and statistical inference and was one of the main reasons for studying the operators in (1.3).

We introduce the time-varying fractionally integrated processes $\left(X_{t}\right)$ and $\left(Y_{t}\right)$ defined by equations

$$
A(-\mathrm{d}) X_{t}=G \varepsilon_{t}, \quad B(-\mathrm{d}) Y_{t}=G \varepsilon_{t},
$$

where $\left(\varepsilon_{t}, t \in \mathbf{Z}\right)$ is a stationary martingale difference sequence and $G$ is a short memory filter with absolutely summable coefficients:

$$
G x_{t}=\sum_{j=0}^{\infty} g_{j} x_{t-j}, \quad \text { with } \quad \sum_{j=0}^{\infty}\left|g_{j}\right|<\infty .
$$

According to (1.6), the solutions of equations (1.7) are defined by

$$
\begin{aligned}
& X_{t}=A(-\mathbf{d})^{-1} G \varepsilon_{t}=B(\mathbf{d}) G \varepsilon_{t}=\sum_{j=0}^{\infty}(b \star g)_{j}(t) \varepsilon_{t-j} \\
& Y_{t}=B(-\mathbf{d})^{-1} G \varepsilon_{t}=A(\mathrm{~d}) G \varepsilon_{t}=\sum_{j=0}^{\infty}(a \star g)_{j}(t) \varepsilon_{t-j}
\end{aligned}
$$

where

$$
(b \star g)_{j}(t):=\sum_{i=0}^{j} b_{i}(t) g_{j-i}, \quad(a \star g)_{j}(t):=\sum_{i=0}^{j} a_{i}(t) g_{j-i}
$$


are the impulse responses of the product operators $B(\mathbf{d}) G, A(\mathbf{d}) G$, respectively. Note that when $d_{t} \equiv d$ is a constant, the processes (1.8) and (1.9) are stationary and contain the class of (stationary) $\operatorname{FARIMA}(p, d, q)$.

The asymptotic results below are proved for sequences $\mathbf{d}=\left(d_{t}, t \in \mathbf{Z}\right)$ such that there exist limits

$$
d_{ \pm}:=\lim _{t \rightarrow \pm \infty} d_{t} \in\left(0, \frac{1}{2}\right)
$$

The limit $d_{+}=\lim _{t \rightarrow \infty} d_{t}$ can be interpreted as the intensity of long memory of $Y_{t}$ and $X_{t}$ in (1.7) in the distant future and $d_{-}=\lim _{t \rightarrow-\infty} d_{t}$ as the corresponding intensity in the distant past. We systematically omit the (tedious) estimation of the covariances $\operatorname{cov}\left(X_{s}, X_{t}\right)$ and $\operatorname{cov}\left(Y_{s}, Y_{t}\right)$. The interested reader is referred to the preprint [15], where long memory behavior of these covariances is established. We focus here on the asymptotics of partial sums and prove that for $\mathbf{d}$ satisfying (1.11), the partial sums processes of $Y_{t}=A(\mathbf{d}) G \varepsilon_{t}$ and $X_{t}=B(\mathbf{d}) G \varepsilon_{t}$ converge to some Gaussian processes which depend on the limits $d_{ \pm}$in (1.11) and the sum $\bar{g}=\sum_{i=1}^{\infty} g_{i}$ alone. More precisely, according to Theorem 5.1, the partial sums process of $Y_{t}=A(\mathrm{~d}) G \varepsilon_{t}$ weakly converges, under normalization $N^{d_{+}+1 / 2}$, to the sum of two independent self-similar processes: a type II fBm and a smooth Gaussian process with asymptotically vanishing increments. On the other hand, as shown in Theorem 5.2, the partial sums process of $X_{t}=B(\mathbf{d}) G \varepsilon_{t}$ converges to different limits depending on whether $d_{+}>d_{-}$or $d_{+}<d_{-}$holds: in the first case, the limit process is a type II fBm, while in the second case, it is a Gaussian process with smooth paths and asymptotically vanishing increments. We note that the last fact is very unusual in the context of stationary processes, where the limit of partial sums has nondifferentiable trajectories as a rule.

It is also interesting to note that, apart from condition (1.11), we do not assume that the sequence $d_{t}$ belongs to $\left(0, \frac{1}{2}\right)$ or to any other a priori fixed interval of the real line. When $d_{t}$ has excursions above $\frac{1}{2}$, the simulated trajectories of processes $X_{t}$ and $Y_{t}$ in (1.8) and (1.9) present sudden «bursts» similar to those encountered in infinite variance processes.

The results of the present paper were recently extended into several directions. In [16], we discuss the case of almost periodic sequences $\mathbf{d}$ and show that, in the last case, «averaging of nonstationary long memory» occurs and the partial sums processes of time-varying filters $Y_{t}$ and $X_{t}$ converge to a usual $\mathrm{fBm}$ with parameter determined by the mean value $\bar{d}$ of the almost periodic function $\mathrm{d}$. A similar behavior of partial sums was established in the case when $\mathbf{d}$ is a random stationary process [9]. The case of time-varying fractionally integrated processes with infinite variance and the convergence of the corresponding partial sums processes was discussed in [4]. In [19] the definition of time-varying fractional operators in (1.3) is extended to 
the continuous time setup, as a limit of discrete operators corresponding to slowly varying sequences of the form $d_{N, t}=d(t / N), t \in \mathbf{Z}$, where $d(x)$, $x \in \mathbf{R}$, is a given function on the real line. This extension is useful for modeling multifractality. See [14] and [1] for an approach to multifractality involving time substitution of a $\mathrm{fBm}$.

The paper is organized as follows. In Section 2 we study the general properties of time-varying filters (1.3), (1.4), and (1.5). Section 3 is devoted to the proof of invertibility (1.6). Section 4 discusses some properties of self-similar processes later obtained in Section 5 as limits of the partial sums. Section 5 formulates and proves the main convergence theorems (Theorems 5.1 and 5.2). Technical lemmas are relegated to Section 6.

2. General properties of time-varying fractional filters. Let $\mathscr{X}^{p}$ $(p \geqslant 1)$ denote the class of all real-valued random processes $\left(x_{t}, t \in \mathbf{Z}\right)$ such that $\sup _{t} \mathbf{E}\left|x_{t}\right|^{p}<\infty$. Let $\mathscr{X}_{0}^{2} \subset \mathscr{X}^{2}$ denote the subclass of all orthogonal sequences $\left(x_{t}, t \in \mathbf{Z}\right)$ with zero mean, i.e., such that $\mathbf{E} x_{t}=0, \mathbf{E} x_{t} x_{s}=0$ for any $t, s \in \mathbf{Z}, t \neq s$. Everywhere in what follows we assume that the sequence $\mathbf{d}=\left(d_{t}, t \in \mathbf{Z}\right)$ is bounded, i.e.,

$$
\sup _{t \in \mathbf{Z}}\left|d_{t}\right|<\infty
$$

$\mathrm{D}$ e $\mathrm{f}$ in it i o $\mathrm{n} 2.1$. Let $D$ be a real number. We say that a sequence $\mathbf{d}=\left(d_{t}, t \in \mathbf{Z}\right)$ satisfies condition $\mathscr{A}(D)$ if there exists $K<\infty$ such that for all integers $s<t$ such that $t-s>K$,

$$
\sum_{s<u<t} \frac{d_{u}-D}{t-u} \leqslant 0 .
$$

Similarly, we say that $\mathbf{d}=\left(d_{t}, t \in \mathbf{Z}\right)$ satisfies condition $\mathscr{B}(D)$ if there exists $K<\infty$ such that for all integers $s<t$ such that $|t-s|>K$,

$$
\sum_{s<u<t} \frac{d_{u}-D}{u-s} \leqslant 0
$$

Clearly, if $\left(d_{t}, t \in \mathbf{Z}\right)$ satisfies condition $\mathscr{A}(D)$ (respectively, condition $\mathscr{B}(D))$, then the sequence $\left(d_{t+\tau}, t \in \mathbf{Z}\right)$ also satisfies condition $\mathscr{A}(D)$ (respectively, condition $\mathscr{B}(D)$ ), for any $\tau \in \mathbf{Z}$; in other words, conditions $\mathscr{A}(D)$ and $\mathscr{B}(D)$ are invariant with respect to translations. It is also obvious that if $\left(d_{t}, t \in \mathbf{Z}\right)$ satisfies condition $\mathscr{A}(D)$ (respectively, $\mathscr{B}(D)$ ), then the time-reversed sequence $\left(d_{-t}, t \in \mathbf{Z}\right)$ satisfies condition $\mathscr{B}(D)$ (respectively, condition $\mathscr{A}(D)$ ). The following example shows that conditions $\mathscr{A}(D)$ and $\mathscr{B}(D)$ are not equivalent.

$\mathrm{Ex} \mathrm{a} \mathrm{m} \mathrm{p} \mathrm{l} \mathrm{e} \mathrm{2.1.} \mathrm{Let}$

$$
d_{t}=\left\{\begin{array}{rlr}
-2, & t=0 \\
1, & t=1 \\
-1, & t=2, \\
0, & t \neq 0,1,2
\end{array}\right.
$$


The sequence $\left(d_{t}, t \in \mathbf{Z}\right)$ satisfies condition $\mathscr{A}(0)$ and does not satisfy condition $\mathscr{B}(0)$. Indeed,

$$
\sum_{s<u<t} \frac{d_{u}}{t-u}= \begin{cases}-\frac{2}{t}+\frac{1}{t-1}-\frac{1}{t-2} \leqslant 0, & s<0, \quad t>2, \\ \frac{1}{t-1}-\frac{1}{t-2} \leqslant 0, & s=0, \quad t>2, \\ -\frac{1}{t-2} \leqslant 0, & s=1, \quad t>2, \\ 0, & s<0, \quad t=2, \\ -2, & s<0, \quad t=1,\end{cases}
$$

and $\mathscr{A}(0)$ holds. On the other hand, $\sum_{0<u<t} d_{u} / u=\frac{1}{2}>0$ for $t>2$ so that $\mathscr{B}(0)$ is not satisfied.

Proposition 2.1. Let $D \notin\{x \in \mathbf{Z}: x \leqslant 0\}$.

(i) Let $\left(d_{t}, t \in \mathbf{Z}\right)$ satisfy condition $\mathscr{A}(D)$. Then there exists a constant $C<\infty$ such that for all $t \in \mathbf{Z}$ and $j \geqslant 1$

$$
\left|a_{j}(t)\right| \leqslant C\left|\psi_{j}(D)\right| \text {. }
$$

(ii) Let $\left(d_{t}, t \in \mathbf{Z}\right)$ satisfy condition $\mathscr{B}(D)$. Then there exists a constant $C<\infty$ such that for all $t \in \mathbf{Z}$ and $j \geqslant 1$

$$
\left|b_{j}(t)\right| \leqslant C\left|\psi_{j}(D)\right| \text {. }
$$

P r o of. (i) Let $j_{0}$ be large enough so that $\frac{1}{2}<\left(d_{u}+k-1\right) /$ $(D+k-1)<\frac{3}{2}$ holds for all $u \in \mathbf{Z}, k>j_{0}$. Then

$$
\begin{aligned}
\Lambda_{j}(t):=\frac{\left|a_{j}(t)\right|}{\left|\psi_{j}(D)\right|} & =C\left(t, j_{0}\right) \prod_{k=j_{0}+1}^{j} \frac{d_{t-k}+k-1}{D+k-1} \\
& =C\left(t, j_{0}\right) \prod_{k=j_{0}+1}^{j}\left(1+\frac{d_{t-k}-D}{D+k-1}\right),
\end{aligned}
$$

where

$$
C\left(t, j_{0}\right)=\left|\prod_{k=1}^{j_{0}} \frac{d_{t-k}+k-1}{D+k-1}\right|
$$

is bounded by a constant $C\left(j_{0}\right)$ uniformly in $t \in \mathbf{Z}$, since $\mathbf{d}$ is bounded. Using the inequality: $1+x \leqslant \mathrm{e}^{x}(x \geqslant-1)$, this implies

$$
\Lambda_{j}(t) \leqslant C\left(j_{0}\right) \exp \left\{\sum_{k=j_{0}+1}^{j} \frac{d_{t-k}-D}{D+k-1}\right\} .
$$

Write

$$
\sum_{k=j_{0}+1}^{j} \frac{d_{t-k}-D}{D+k-1}=\sum_{k=1}^{j} \frac{d_{t-k}-D}{k}+R
$$


where $\sum_{k=1}^{j}\left(d_{t-k}-D\right) / k \leqslant 0$ by condition $\mathscr{A}(D)$, provided $j$ is large enough. Next,

$$
R:=\sum_{k=j_{0}+1}^{j}\left(d_{t-k}-D\right)\left(\frac{1}{D+k-1}-\frac{1}{k}\right)-\sum_{k=1}^{j_{0}} \frac{d_{t-k}-D}{k}=: R^{\prime}-R^{\prime \prime},
$$

where $\left|R^{\prime}\right|$ and $\left|R^{\prime \prime}\right|$ are bounded uniformly in $t \in \mathbf{Z}$ and $j>j_{0}$. This proves $\Lambda_{j}(t) \leqslant C$ uniformly in $t, j$.

(ii) We omit this proof which is similar to the proof of (i).

Corollary 2.1. Let $\mathbf{d}$ satisfy condition $\mathscr{A}(D)$ (respectively, condition $\mathscr{B}(D))$. Moreover, let there exist $C, \delta>0$ such that for any $t \in \mathbf{Z}$, $j \geqslant 0$

$$
\left|g_{j}\right| \leqslant C j^{-1-\delta} .
$$

(i) If $D<0$, then $A(\mathbf{d}) G$ (respectively, $B(\mathrm{~d}) G$ ) is a bounded operator $\mathscr{X}^{1} \rightarrow \mathscr{X}^{1}$.

(ii) If $D<\frac{1}{2}$, then $A(\mathbf{d}) G$ (respectively, $B(\mathbf{d}) G$ ) is a bounded operator $\mathscr{X}_{0}^{2} \rightarrow \mathscr{X}^{2}$.

P r o o f. (i) It suffices to show that there exist $C^{\prime}, \delta^{\prime}>0$ such that for all $t \in \mathbf{Z}, j \geqslant 0$

$$
\left|(a \star g)_{j}(t)\right| \leqslant C^{\prime} j^{-1-\delta^{\prime}}, \quad\left|(b \star g)_{j}(t)\right| \leqslant C^{\prime}(1+j)^{-1-\delta^{\prime}} .
$$

In view of Proposition 2.1 and well-known properties of FARIMA coefficients in (1.2), inequalities (2.2) follow from the elementary bound:

$$
\sum_{i=0}^{j}(1+i)^{D-1}(1+j-i)^{-1-\delta} \leqslant C^{\prime}(1+j)^{-1-\delta^{\prime}},
$$

where $\delta^{\prime}=\min (\delta,-D)>0$.

(ii) Follows by the same argument and (2.3), where $\delta^{\prime}=-D>-\frac{1}{2}$.

Proposition 2.2. $\quad$ (i) A sequence $\mathrm{d}$ satisfies conditions $\mathscr{A}(D)$ and $\mathscr{B}(D)$ for any $D$ such that

$$
D>\limsup _{h \rightarrow+\infty}\left(\frac{1}{h} \sup _{s \in \mathbf{Z}} \sum_{s<u<s+h} d_{u}\right)=: \bar{d} .
$$

(ii) A sequence $\mathbf{d}$ satisfies conditions $\mathscr{A}(D)$ and $\mathscr{B}(D)$ for any $D>$ $\lim \sup _{|t| \rightarrow \infty} d_{t}$.

P r o of. (i) Let $\delta>0$ and $D^{\prime}=\bar{d}+\delta, D^{\prime \prime}=\bar{d}+2 \delta$. According to the definition, for any $\delta>0$ there exists $K<\infty$ such that for all $s<t$, $t-s>K$,

$$
\alpha_{s, t}:=\sum_{s<u<t}\left(d_{u}-D^{\prime}\right)<0
$$


Then, for all $s<t, t-s>K$, we have

$$
\sum_{s<u<t} \frac{d_{u}-D^{\prime \prime}}{t-u}=-\delta \sum_{s<u<t} \frac{1}{t-u}+\sum_{s<u<t} \frac{d_{u}-D^{\prime}}{t-u}=:-\delta J_{s, t}^{\prime}+J_{s, t}^{\prime \prime},
$$

where $J_{s, t}^{\prime} \rightarrow \infty$ as $t-s \rightarrow \infty$. On the other hand, using (2.4) and summation by parts,

$$
J_{s, t}^{\prime \prime}=\frac{\alpha_{s, t}}{t-s-1}+\sum_{i=1}^{t-s-2} \alpha_{t-i-1, t}\left(\frac{1}{i}-\frac{1}{i+1}\right)<\sum_{i=1}^{t-s-2} \frac{\alpha_{t-i-1, t}}{i(i+1)}=: I_{s, t},
$$

where

$$
I_{s, t}=\sum_{i=1}^{K} \frac{\alpha_{t-i-1, i}}{i(i+1)}+\sum_{i=K+1}^{t-s-2} \frac{\alpha_{t-i-1, i}}{i(i+1)}=: I_{s, t}^{\prime}+I_{s, t}^{\prime \prime}
$$

and where $\left|I_{s, t}^{\prime}\right| \leqslant C$ is uniformly bounded in $t$ for any $K<\infty$ fixed while $I_{s, t}^{\prime \prime}<0$ by (2.4). This proves condition $\mathscr{A}(D)$ with any $D>\bar{d}$. The proof of condition $\mathscr{B}(D)$ follows similarly.

(ii) Follows from (i) and the fact that $\bar{d} \leqslant \lim \sup _{|t| \rightarrow \infty} d_{t}$.

3. Invertibility. In this section, we prove that, for a sequence $\mathbf{d}$ such that the two operators are well defined, $A(\mathbf{d})$ and $B(-\mathbf{d})$ are inverse to each other.

Theorem 3.1. (i) Let a sequence $\mathbf{d}=\left(d_{t}, t \in \mathbf{Z}\right)$ satisfy condition $\mathscr{A}\left(D_{0}\right)$ with $D_{0}<\frac{1}{2}$, and let the sequence $-\mathbf{d}=\left(-d_{t}, t \in \mathbf{Z}\right)$ satisfy condition $\mathscr{B}\left(D_{1}\right)$ with $D_{1}<0$. Then $A(\mathbf{d})$ maps $\mathscr{X}_{0}^{2}$ to $\mathscr{X}^{2} \subset \mathscr{X}^{1}, B(-\mathbf{d})$ maps $\mathscr{X}^{1}$ to itself, and

$$
B(-\mathrm{d}) \circ A(\mathbf{d})=I
$$

where $I$ is the identity operator on $\mathscr{X}_{0}^{2}$.

(ii) Let a sequence $\mathbf{d}=\left(d_{t}, t \in \mathbf{Z}\right)$ satisfy condition $\mathscr{B}\left(D_{2}\right)$ with $D_{2}<\frac{1}{2}$, and let the sequence $-\mathbf{d}=\left(-d_{t}, t \in \mathbf{Z}\right)$ satisfy condition $\mathscr{A}\left(D_{3}\right)$ with $D_{3}<0$. Then $B(\mathbf{d})$ maps $\mathscr{X}_{0}^{2}$ to $\mathscr{X}^{2} \subset \mathscr{X}^{1}, A(-\mathbf{d})$ maps $\mathscr{X}^{1}$ to itself, and

$$
A(-\mathrm{d}) \circ B(\mathrm{~d})=I \text {. }
$$

As an illustration of this theorem, consider sequences $\mathbf{d}$ having limits $d_{ \pm} \in\left(0, \frac{1}{2}\right)$ at $\pm \infty$. Then Theorem 3.1 together with Proposition 2.2 imply that $A(-\mathbf{d}) \circ B(\mathbf{d})$ and $B(-\mathbf{d}) \circ A(\mathbf{d})$ both are the identity operator on the space $\mathscr{X}_{0}^{2}$ of all $L^{2}$ zero-mean orthogonal sequences.

P r o of of The or e m 3.1. The first parts of (i) and (ii) are directly deduced from Corollary 2.1. Let us prove (3.1) and (3.2).

To prove (3.1), it is enough to show that for every $n \geqslant 1$

$$
\Delta_{n}(t)=\sum_{j=0}^{n} b_{j}^{-}(t) a_{n-j}(t-j)=0
$$


where

$$
b_{j}^{-}(t)=(-1)^{j} \frac{d_{t-1}}{j !} \prod_{k=1}^{j-1}\left(d_{t-k-1}-j+k\right), \quad j \geqslant 1,
$$

are the coefficients defined in (1.5), with $\mathbf{d}$ replaced by $-\mathrm{d}$.

We obtain

$$
\begin{aligned}
\Delta_{n}(t)= & a_{n}(t)+b_{1}^{-}(t) a_{n-1}(t-1)+\sum_{j=2}^{n-1} b_{j}^{-}(t) a_{n-j}(t-j)+b_{n}^{-}(t) \\
= & \frac{1}{n !} \prod_{k=1}^{n}\left(d_{t-k}+k-1\right)-\frac{d_{t-1}}{(n-1) !} \prod_{k=2}^{n}\left(d_{t-k}+k-2\right) \\
& +d_{t-1} \sum_{j=2}^{n-1} \frac{(-1)^{j}}{j !(n-j) !} \prod_{k=1}^{j-1}\left(d_{t-k-1}+k-j\right) \prod_{\ell=1}^{n-j}\left(d_{t-j-\ell}+\ell-1\right) \\
& +\frac{d_{t-1}(-1)^{n}}{n !} \prod_{k=1}^{n-1}\left(d_{t-1-k}+k-n\right) .
\end{aligned}
$$

Next,

$$
\prod_{k=1}^{j-1}\left(d_{t-k-1}+k-j\right) \prod_{\ell=1}^{n-j}\left(d_{t-j-\ell}+\ell-1\right)=\prod_{k=1}^{n-1}\left(d_{t-k-1}+k-j\right)
$$

leads to

$$
\Delta_{n}(t)=\frac{d_{t-1}}{n !} \sum_{j=0}^{n}(-1)^{j}\left(\begin{array}{c}
n \\
j
\end{array}\right)\left(y_{1}+n-j\right) \cdots\left(y_{n-1}+n-j\right)
$$

with $y_{k}:=d_{t-k-1}-n+k, k=1, \ldots, n-1$. The result is now deduced from Lemma 3.1 below.

To prove (3.2), it is enough to show that for every $n \geqslant 1$

$$
\tilde{\Delta}_{n}(t):=\sum_{j=0}^{n} a_{j}^{-}(t) b_{n-j}(t-j)=0
$$

where the coefficients $a_{j}^{-}(t)$ are defined by (1.4) with $\left(d_{t}, t \in \mathbf{Z}\right)$ replaced by $\left(-d_{t}, t \in \mathbf{Z}\right)$.

Using (1.4) and (1.5), for $j=1, \ldots, n-2$ we have

$$
a_{j}^{-}(t) b_{n-j}(t-j)=\frac{d_{t-1}}{j !(n-j) !} \prod_{k=2}^{j}\left(k-1-d_{t-k}\right) d_{t-j-1} \prod_{\ell=1}^{n-j-1}\left(\ell+d_{t-n+\ell-1}\right) \text {. }
$$

Using the notation $x_{k}:=d_{t-n+k-1}+k, k=1, \ldots, n-1$, the last product can be rewritten as

$$
\begin{aligned}
& \prod_{k=2}^{j}\left(k-1-d_{t-k}\right) d_{t-j-1} \prod_{\ell=1}^{n-j-1}\left(\ell+d_{t-n+\ell-1}\right) \\
& =\left(\prod_{\ell=1}^{n-j-1} x_{\ell}\right)\left(n-j-x_{n-j}\right) \prod_{\ell=n-j+1}^{n-1}\left(n-x_{\ell}\right) .
\end{aligned}
$$


Similarly,

$$
\begin{aligned}
a_{0}^{-}(t) b_{n}(t) & =\frac{d_{t-1}}{n !} \prod_{\ell=1}^{n-1} x_{\ell} \\
a_{n-1}^{-}(t) b_{1}(t-n+1) & =\frac{d_{t-1}}{(n-1) !}\left(1-x_{1}\right) \prod_{\ell=2}^{n-1}\left(n-x_{\ell}\right), \\
a_{n}^{-}(t) b_{0}(t-n) & =\frac{d_{t-1}}{n !}(-1) \prod_{\ell=1}^{n-1}\left(n-x_{\ell}\right) .
\end{aligned}
$$

This yields

$$
\begin{aligned}
\tilde{\Delta}_{n}(t)=\frac{d_{t-1}}{n !} & {\left[\prod_{\ell=1}^{n-1} x_{\ell}+\sum_{j=1}^{n-2}\left(\begin{array}{c}
n \\
j
\end{array}\right)\left(\prod_{\ell=1}^{n-j-1} x_{\ell}\right)\left(n-j-x_{n-j}\right) \prod_{\ell=n-j+1}^{n-1}\left(n-x_{\ell}\right)\right.} \\
& \left.+\left(\begin{array}{c}
n \\
n-1
\end{array}\right)\left(1-x_{1}\right) \prod_{\ell=2}^{n-1}\left(n-x_{\ell}\right)-\prod_{\ell=1}^{n-1}\left(n-x_{\ell}\right)\right]=: \frac{d_{t-1}}{n !} \sum_{j=0}^{n} A_{j}
\end{aligned}
$$

We claim that for any $m=1, \ldots, n-2$

$$
\sum_{j=0}^{m} A_{j}=\left(\begin{array}{c}
n-1 \\
m
\end{array}\right)\left(\prod_{\ell=1}^{n-m-1} x_{\ell}\right) \prod_{k=n-m}^{n-1}\left(n-x_{k}\right) .
$$

Indeed, for $m=1$,

$$
\begin{aligned}
A_{0}+A_{1} & =\prod_{\ell=1}^{n-1} x_{\ell}+\left(\begin{array}{c}
n \\
1
\end{array}\right)\left(\prod_{\ell=1}^{n-2} x_{\ell}\right)\left(n-1-x_{n-1}\right) \\
& =\left(\begin{array}{c}
n-1 \\
1
\end{array}\right)\left(\prod_{\ell=1}^{n-2} x_{\ell}\right)\left(n-x_{n-1}\right) .
\end{aligned}
$$

The induction step $m-1 \rightarrow m$ easily follows:

$$
\begin{aligned}
\sum_{j=0}^{m} A_{j}= & \left(\begin{array}{c}
n-1 \\
m-1
\end{array}\right)\left(\prod_{\ell=1}^{n-m} x_{\ell}\right) \prod_{k=n-m+1}^{n-1}\left(n-x_{k}\right) \\
& +\left(\begin{array}{c}
n \\
m
\end{array}\right)\left(\begin{array}{c}
n-m-1 \\
\prod_{\ell=1}^{n} x_{\ell}
\end{array}\right)\left(n-m-x_{n-m}\right) \prod_{k=n-m+1}^{n-1}\left(n-x_{k}\right) \\
= & \left(\begin{array}{c}
n-1 \\
m
\end{array}\right)\left(\prod_{\ell=1}^{n-m} x_{\ell}\right) \prod_{k=n-m}^{n-1}\left(n-x_{k}\right) .
\end{aligned}
$$

This proves (3.5). Using it, we obtain

$$
\sum_{j=1}^{n-1} A_{j}=\left(\begin{array}{l}
n-1 \\
n-2
\end{array}\right) x_{1} \prod_{k=2}^{n-1}\left(n-x_{k}\right)+\left(\begin{array}{c}
n \\
n-1
\end{array}\right)\left(1-x_{1}\right) \prod_{k=2}^{n-1}\left(n-x_{k}\right)=-A_{n} .
$$

This proves (3.4) and Theorem 3.1 too. 
Let $n, k \geqslant 1$ be integers. Introduce the polynomial $P_{n, k}\left(x_{1}, \ldots, x_{k}\right)$ in $x_{1}, \ldots, x_{k}$ by

$$
P_{n, k}\left(x_{1}, \ldots, x_{k}\right):=\sum_{j=0}^{n}(-1)^{n-j}\left(\begin{array}{c}
n \\
j
\end{array}\right)\left(j+x_{1}\right) \cdots\left(j+x_{k}\right) .
$$

Lemma 3.1. For every $k \in\{1, \ldots, n\}$, the polynomial function defined in (3.6) satisfies

$$
P_{n, k}\left(x_{1}, \ldots, x_{k}\right) \equiv 0 \text {. }
$$

$\mathrm{P}$ r o o f. For $k<n$, it can easily be checked that

$$
\begin{aligned}
P_{n+1, k}\left(x_{1}, \ldots, x_{k}\right)= & P_{n, k}\left(x_{1}+1, \ldots, x_{k}+1\right)-P_{n, k}\left(x_{1}, \ldots, x_{k}\right), \\
P_{n+1, k+1}\left(x_{1}, \ldots, x_{k+1}\right)= & (n+1) P_{n, k}\left(x_{1}+1, \ldots, x_{k}+1\right) \\
& +x_{k+1} P_{n+1, k}\left(x_{1}, \ldots, x_{k}\right) .
\end{aligned}
$$

First, $P_{2,1}(x)=(2+x)-2(1+x)+x \equiv 0$. Then the lemma is proved by induction on $n$. Assume that $P_{n, k}\left(x_{1}, \ldots, x_{k}\right) \equiv 0$ for some $n \geqslant 1$ and all $1 \leqslant k<n$. Then from (3.7), we deduce that $P_{n+1, k}\left(x_{1}, \ldots, x_{k}\right) \equiv 0$ for all $1 \leqslant k<n$. Finally, (3.8) ensures that $P_{n+1, n}\left(x_{1}, \ldots, x_{n}\right) \equiv 0$, or the validity of the induction step $n \rightarrow n+1$.

$\mathrm{R} \mathrm{e} \mathrm{ma} \mathrm{r} 3.1$. Lemma 3.1 is not true for $k \geqslant n$. In fact, for $k \geqslant$ $n \geqslant 1$,

$$
\frac{1}{n !} P_{n, k}(0, \ldots, 0)=\frac{1}{n !} \sum_{j=0}^{n}(-1)^{n-j}\left(\begin{array}{l}
n \\
j
\end{array}\right) j^{k}=\left\{\begin{array}{l}
k \\
n
\end{array}\right\}
$$

is the Stirling number, or the number of ways $k$ different objects can be partitioned into $n$ groups.

4. A class of self-similar processes with asymptotically stationary or asymptotically vanishing increments.

D ef in it i o n 4.1. Let $W=(W(\tau), \tau \geqslant 0)$ be a stochastic process. We say that

(i) $W$ is self-similar with index $H>0$ ( $H$-self-similar for short) if, for any $a>0$,

$$
(W(a \tau), \tau \geqslant 0)=_{\text {fdd }}\left(a^{H} W(\tau), \tau \geqslant 0\right),
$$

where the subscript «fdd» means that the property holds for finite dimensional distributions;

(ii) $W$ has stationary increments if, for any $T>0$,

$$
(W(T+\tau)-W(T), \tau \geqslant 0)=\text { fdd }(W(\tau)-W(0), \tau \geqslant 0) ;
$$

(iii) $W$ has asymptotically stationary increments if as $T$ goes to $+\infty$

$$
(W(T+\tau)-W(T), \tau \geqslant 0) \longrightarrow_{\text {fdd }}(\widetilde{W}(\tau), \tau \geqslant 0) .
$$

where $\widetilde{W}$ is a nontrivial stochastic process; 
(iv) $W$ has asymptotically vanishing increments if (4.1) holds with $\widetilde{W}(\tau) \equiv 0$.

It is easy to show that if a process $W$ has asymptotically stationary increments (respectively, is $H$-self-similar), then the limit process $\widetilde{W}$ in (4.1) has stationary increments (respectively, is $H$-self-similar). The characterization of all Gaussian self-similar processes with asymptotically stationary (asymptotically vanishing) increments seems to be an interesting problem. One may expect also that if a process $W$ has asymptotically stationary increments and the process $\left(K_{a}^{-1} W(a \tau), \tau \geqslant 0\right)$ tends to some limit process $Q$ as $a \rightarrow \infty$, in the sense of weak convergence of finite dimensional distributions, under some (nonrandom) normalization $K_{a} \rightarrow \infty$, then $Q$ is $H$-self-similar with some $H>0$ and also has stationary increments. The last fact is true and is well known (due to [11]) if $W$ is assumed to have stationary increments.

Let

$$
\begin{aligned}
W_{d}^{+}(\tau) & :=\int_{0}^{\tau} Z(\mathrm{~d} s) \int_{s}^{\tau}(t-s)^{d-1} \mathrm{~d} t \\
U_{d_{+}, d_{-}}(\tau) & :=\int_{-\infty}^{0} Z(\mathrm{~d} s) \int_{0}^{\tau} t^{d_{+}-d_{-}}(t-s)^{d_{-}-1} \mathrm{~d} t \\
V_{d_{+}, d_{-}}(\tau) & :=\int_{-\infty}^{0}(-s)^{d_{-}-d_{+}} Z(\mathrm{~d} s) \int_{0}^{\tau}(t-s)^{d_{+}-1} \mathrm{~d} t
\end{aligned}
$$

where $\left(d, d_{+}, d_{-}\right) \in\left(0, \frac{1}{2}\right)^{3}$ and $Z(\mathrm{~d} s)$ is a standard Gaussian white noise, with zero mean and variance $\mathrm{d} s$. The process $W_{d}^{+}$is called a type II $\mathrm{fBm}$ (see [13]). Note that $W_{d}^{+}$is independent of $U_{d_{+}, d_{-}}$and $V_{d_{+}, d_{-}}$, that $U_{d, d} \equiv V_{d, d}$, and that

$$
W_{d}^{+}(\tau)+U_{d, d}(\tau)=W_{d+1 / 2}(\tau) \quad(\tau \geqslant 0)
$$

is a $\mathrm{fBm}$ with the self-similarity (or Hurst) index $H=d+\frac{1}{2}$.

Proposition 4.1. Let $d, d_{+}, d_{-} \in\left(0, \frac{1}{2}\right)$. Then

(i) The processes $W_{d}^{+}, U_{d_{+}, d_{-}}$, and $V_{d_{+}, d_{-}}$are well defined; they are selfsimilar with respective indices $d+\frac{1}{2}, d_{+}+\frac{1}{2}$, and $d_{-}+\frac{1}{2}$;

(ii) the processes $U_{d_{+}, d_{-}}$and $V_{d_{+}, d_{-}}$have asymptotically vanishing increments, while $W_{d}^{+}$has asymptotically stationary increments tending to those of a $\mathrm{fBm} W_{d+1 / 2}$ in (4.5);

(iii) trajectories of $U_{d_{+}, d_{-}}$and $V_{d_{+}, d_{-}}$are a.s. infinitely differentiable on $(0, \infty)$.

The proof of the above proposition can be found in Appendix (Section 6).

5. Convergence of partial sums processes. Let $d$ be a sequence such that condition (1.11) holds, i.e., we assume the existence of the limits 
$d_{ \pm} \in\left(0, \frac{1}{2}\right)$ at $\pm \infty$. Moreover, we shall assume that $d_{t} \notin \mathbf{Z}$ for all $t$ and that there exists a $\delta>0$ such that

$$
d_{t}=d_{ \pm}+O\left(|t|^{-\delta}\right) \quad(t \rightarrow \pm \infty) .
$$

We also assume that the short-memory filter $G$ satisfies condition (2.1) and, moreover,

$$
\bar{g}:=\sum_{j=0}^{\infty} g_{j} \neq 0
$$

Note that it is without loss of generality that the exponents in (5.1) and in (2.1) are the same. By Proposition 2.2 (ii), condition (1.11) implies both conditions $\mathscr{A}(D)$ and $\mathscr{B}(D)$ with any $D>\max \left(d_{+}, d_{-}\right)$so that the processes $X_{t}$ and $Y_{t}$ in (1.8) and (1.9) are well defined.

The first question to study concerns the asymptotic behavior of the coefficients $a_{v-u}(v)$ and $b_{v-u}(v)$ as $v$ and $v-u$ tend to infinity. In the simple case when the sequence $\mathbf{d}$ assumes two values,

$$
d_{t}= \begin{cases}d_{+}, & t \geqslant 0 \\ d_{-}, & t<0\end{cases}
$$

the filter coefficients $a_{t-s}(t)$ and $b_{t-s}(t)$ can be obtained explicitly from definitions (1.4) and (1.5):

$$
a_{t-s}(t)= \begin{cases}\psi_{t-s}\left(d_{+}\right), & 0 \leqslant s \leqslant t, \\ \psi_{t-s}\left(d_{-}\right) \frac{\psi_{t}\left(d_{+}\right)}{\psi_{t}\left(d_{-}\right)}, & s<0 \leqslant t\end{cases}
$$

and

$$
b_{t-s}(t)= \begin{cases}\psi_{t-s}\left(d_{+}\right), & 0 \leqslant s \leqslant t, \\ \psi_{t-s}\left(d_{+}\right) \frac{d_{+} \psi_{1-s}\left(d_{-}\right)}{d_{-} \psi_{1-s}\left(d_{+}\right)}, & s<0 \leqslant t .\end{cases}
$$

It turns out that these relations remain valid asymptotically also in the general case of $\mathbf{d}$, as stated in Lemma 6.1 in Appendix (Section 6).

The presence of the short-memory filter $G$ preserves this asymptotic behavior up to a multiplicative constant, as stated below.

Lemma 5.1. Let $\left(d_{t}, t \in \mathbf{Z}\right)$ be a sequence such that (1.11) and (5.1) hold. Assume that the short memory filter $G$ satisfies (2.1) and (5.2). Then there exist $C, \delta_{1}>0$ independent of $s, t$ and such that

$$
\begin{aligned}
& (a \star g)_{t-s}(t)=\left\{\begin{array}{lr}
\bar{g} \psi_{t-s}\left(d_{+}\right)+\Theta_{t, s}^{(1)}, & 0 \leqslant s<t, \\
\bar{g} \psi_{t-s}\left(d_{-}\right) \frac{\psi_{t}\left(d_{+}\right)}{\psi_{t}\left(d_{-}\right)}+\Theta_{t, s}^{(1)}, & s<0 \leqslant t,
\end{array}\right. \\
& (b \star g)_{t-s}(t)=\left\{\begin{array}{lr}
\bar{g} \psi_{t-s}\left(d_{+}\right)+\Theta_{t, s}^{(2)}, & 0 \leqslant s<t, \\
\bar{g} \psi_{t-s}\left(d_{+}\right) \frac{d_{+} \psi_{1-s}\left(d_{-}\right)}{d_{-} \psi_{1-s}\left(d_{+}\right)}+\Theta_{t, s}^{(2)}, & s<0 \leqslant t,
\end{array}\right.
\end{aligned}
$$


where

$$
\begin{aligned}
\left|\Theta_{t, s}^{(1)}\right| \leqslant & C\left(|t-s|^{-\delta_{1}}+|s|^{-\delta_{1}}+|t|^{-\delta_{1}}\right) \\
& \times\left\{\begin{array}{lc}
(t-s)^{d_{+}-1}, & 0 \leqslant s<t, \\
t^{d_{+}-d_{-}}(t-s)^{d_{-}-1}, & s<0 \leqslant t,
\end{array}\right. \\
\left|\Theta_{t, s}^{(2)}\right| \leqslant & C\left(|t-s|^{-\delta_{1}}+|s|^{-\delta_{1}}+|t|^{-\delta_{1}}\right) \\
& \times \begin{cases}(t-s)^{d_{+}-1}, & 0 \leqslant s<t, \\
|s|^{\left(d_{-}-d_{+}\right) \vee 0}(t-s)^{d_{+}-1}, & s<0 \leqslant t .\end{cases}
\end{aligned}
$$

The proof of this lemma is given in Appendix (Section 6).

In the sequel, $\longrightarrow_{D[0,1]}$ denotes the weak convergence of random elements in the Skorokhod space $D[0,1]$ endowed with the sup-topology.

Theorem 5.1. Let $Y_{t}:=A(\mathbf{d}) G \varepsilon_{t}$, where $\left(\varepsilon_{t}, t \in \mathbf{Z}\right)$ is a stationary ergodic martingale difference sequence, with unit variance. Moreover, assume that the assumptions of Lemma 5.1 hold. Then

$$
N^{-d_{+}-(1 / 2)} \sum_{t=1}^{[N \tau]} Y_{t} \longrightarrow D[0,1] \frac{\bar{g}}{\Gamma\left(d_{+}\right)}\left(W_{d_{+}}^{+}(\tau)+U_{d_{+}, d_{-}}(\tau)\right)
$$

where $W_{d}^{+}(\tau)$ and $U_{d_{+}, d_{-}}(\tau)$ are defined in (4.2) and (4.3).

$\mathrm{Pr}$ o o f. To prove the convergence of finite dimensional distributions, we use the so-called scheme of discrete stochastic integrals [17]. We shall restrict the proof to the case $\tau=1$. Let $I_{N}$ stand for the left-hand side of (5.7), where $\tau=1$. We shall write $I_{N}$ as a discrete stochastic integral with respect to a stochastic measure with orthogonal increments. Namely,

$$
I_{N}=\int_{\mathbf{R}} f_{N}(x) Z_{N}(\mathrm{~d} x)
$$

where the piecewise constant function $f_{N}=f_{N}(x), x \in \mathbf{R}$, is defined by

$$
f_{N}(x):=N^{-d_{+}} \begin{cases}\sum_{t=1}^{N}(a \star g)_{t-s}(t), & x \in\left(\frac{s-1}{N}, \frac{s}{N}\right], \quad x \in(-\infty, 1], \\ 0, & \text { otherwise. }\end{cases}
$$

The stochastic measure $Z_{N}$ is defined on finite intervals $\left(x^{\prime}, x^{\prime \prime}\right], x^{\prime}<x^{\prime \prime}$, by

$$
Z_{N}\left(\left(x^{\prime}, x^{\prime \prime}\right]\right):=N^{-1 / 2} \sum_{x^{\prime}<s / N \leqslant x^{\prime \prime}} \varepsilon_{s} .
$$

The discrete stochastic integral $\int_{\mathbf{R}} q(x) Z_{N}(\mathrm{~d} x) \equiv \int q \mathrm{~d} Z_{N}$ is defined by $\sum_{s} q_{s} Z_{N}\left(\Delta_{s}\right)$ for each simple function $q(x)$ taking nonzero constant values $q_{s}$ on a finite number of intervals $\Delta_{s}=(s / N,(s+1) / N], s \in \mathbf{Z}$, and 
vanishing elsewhere. Because of the orthogonality of the measure $Z_{N}$ (which is a consequence of the martingale difference property of the $\varepsilon_{s}$ 's),

$$
\mathbf{E}\left(\int q \mathrm{~d} Z_{N}\right)^{2}=\|q\|_{2}^{2}=\int_{\mathbf{R}} q^{2}(x) \mathrm{d} x .
$$

According to the classical martingale central limit theorem [2], for any $m<\infty$ and any disjoint intervals $\left(x_{i}^{\prime}, x_{i}^{\prime \prime}\right], i=1, \ldots, m$,

$$
\left(Z_{N}\left(\left(x_{1}^{\prime}, x_{1}^{\prime \prime}\right]\right), \ldots, Z_{N}\left(\left(x_{m}^{\prime}, x_{m}^{\prime \prime}\right]\right)\right) \longrightarrow_{\text {law }}\left(Z\left(\left(x_{1}^{\prime}, x_{1}^{\prime \prime}\right]\right), \ldots, Z\left(\left(x_{m}^{\prime}, x_{m}^{\prime \prime}\right]\right)\right)
$$

where $Z(\mathrm{~d} x)$ is a standard Gaussian noise with mean zero and variance $\mathrm{d} x$. The right-hand side of (5.7) (corresponding to $\tau=1$ ) is written as $I=$ $\int_{\mathbf{R}} f(x) Z(\mathrm{~d} x) \equiv \int f \mathrm{~d} Z$, where

$$
f(x):=\frac{\bar{g}}{\Gamma\left(d_{+}\right)} \begin{cases}\int_{0}^{1}(t-x)_{+}^{d_{+}-1} \mathrm{~d} t, & x \in[0,1] \\ \int_{0}^{1}(t-x)^{d_{-}-1} t^{d_{+}-d_{-}} \mathrm{d} t, & x \in(-\infty, 0) \\ 0, & \text { otherwise. }\end{cases}
$$

Then convergence $I_{N} \rightarrow$ law $I$ follows from (5.8)-(5.11) and from

$$
\left\|f_{N}-f\right\|_{2} \rightarrow 0 \quad(N \rightarrow \infty) ;
$$

see [17] for details. With Lemma 5.1 in mind, set

$$
\tilde{f}_{N}(x):=\bar{g} N^{-d_{+}} \begin{cases}\sum_{t=1}^{N} \psi_{t-s}\left(d_{+}\right), & x \in\left(\frac{s-1}{N}, \frac{s}{N}\right], x \in[0,1], \\ \sum_{t=1}^{N} \psi_{t-s}\left(d_{-}\right) \frac{\psi_{t}\left(d_{+}\right)}{\psi_{t}\left(d_{-}\right)}, & x \in\left(\frac{s-1}{N}, \frac{s}{N}\right], x \in(-\infty, 0), \\ 0, & \text { otherwise. }\end{cases}
$$

Clearly the convergence (5.12) follows from

$$
\left\|\tilde{f}_{N}-f_{N}\right\|_{2} \rightarrow 0 \quad(N \rightarrow \infty)
$$

and from

$$
\tilde{f}_{N}(x) \rightarrow f(x), \quad\left|\tilde{f}_{N}(x)\right| \leqslant \bar{f}(x),
$$

where $\bar{f} \in L^{2}(\mathbf{R})$.

By Lemma 5.1, (5.3), (5.5), and the dominated convergence theorem, $\tilde{f}_{N}(x)-f_{N}(x)$ tends to zero almost everywhere. Indeed, since

$$
\delta(t, s):=|t-s|^{-\delta_{1}}+|s|^{-\delta_{1}}+|t|^{-\delta_{1}}
$$


tends to 0 as $t \rightarrow \infty, s \rightarrow-\infty$ and is uniformly bounded in $s<t$, for any $x<0, x \in((s-1) / N, s / N]$, we obtain

$$
\begin{aligned}
\left|\tilde{f}_{N}(x)-f_{N}(x)\right| & \leqslant C N^{-d_{+}} \sum_{t=1}^{N}|t-s|^{d_{-}-1} \frac{t^{d_{+}-1}}{t^{d_{-}-1}} \delta(t, s) \\
& \leqslant C N^{-d_{+}} \int_{0}^{N}(t-N x)^{d_{--1}} t^{d_{+}-d_{-}} \delta([t],[N x]) \mathrm{d} t \\
& =C \int_{0}^{1}(\tau-x)^{d_{-}-1} \tau^{d_{+}-d_{-}} \delta([N \tau],[N x]) \mathrm{d} \tau=o(1)
\end{aligned}
$$

and the case $0<x<1$ follows similarly. From Lemma 5.1, (5.3), (5.5), it also follows that $\left|\tilde{f}_{N}(x)-f_{N}(x)\right| \leqslant C \tilde{f}_{N}(x)$, with $C<\infty$ independent of $N, x$, and therefore (5.13) is a consequence of (5.14) and the dominated convergence theorem. In turn, relations (5.14) can easily be shown using well-known properties of FARIMA $(0, d, 0)$ weights $\psi_{t}(d)$. This proves finite dimensional convergence in (5.7).

Let us check the tightness in $D[0,1]$. By a well-known criterion, it suffices to show that there exists $\gamma>0$ such that for $0 \leqslant \tau<\tau+h \leqslant 1$

$$
\mathbf{E}\left(\sum_{s=[N \tau]}^{[N(\tau+h)]} Y_{s}\right)^{2} \leqslant C h^{1+\gamma} N^{1+2 d_{+}} .
$$

Using Lemma 5.1, the proof of (5.15) reduces to

$$
\begin{aligned}
& \sum_{s=1}^{N} \sum_{t_{1}, t_{2}=[N \tau]}^{[N(\tau+h)]}\left|\psi_{t_{1}-s}\left(d_{+}\right) \psi_{t_{2}-s}\left(d_{+}\right)\right| \leqslant C h^{1+\gamma} N^{1+2 d_{+}}, \\
& \sum_{s=-\infty}^{0} \sum_{t_{1}, t_{2}=[N \tau]}^{[N(\tau+h)]}\left|\psi_{t_{1}-s}\left(d_{-}\right) \psi_{t_{2}-s}\left(d_{-}\right) \frac{\psi_{t_{1}}\left(d_{+}\right)}{\psi_{t_{1}}\left(d_{-}\right)} \frac{\psi_{t_{2}}\left(d_{+}\right)}{\psi_{t_{2}}\left(d_{-}\right)}\right| \\
& \quad \leqslant C h^{1+\gamma} N^{1+2 d_{+}}
\end{aligned}
$$

Let us check (5.17); the proof of (5.16) follows similarly and is omitted.

By using integral approximation and the elementary inequality:

$$
\sum_{s \in \mathbf{Z}}\left|t_{1}-s\right|_{+}^{-\alpha}\left|t_{2}-s\right|_{+}^{-\beta} \leqslant C\left|t_{1}-t_{2}\right|_{+}^{1-\alpha-\beta}
$$

which is valid for any $0<\alpha<1,0<\beta<1, \alpha+\beta>1$ and any $t_{1}, t_{2} \in \mathbf{Z}$, inequality (5.17) follows from

$$
\int_{\tau}^{\tau+h} \int_{\tau}^{\tau+h}\left|u_{1}-u_{2}\right|^{2 d_{--1}} u_{1}^{d_{+}-d_{-}} u_{2}^{d_{+-d_{-}}} \mathrm{d} u_{1} \mathrm{~d} u_{2} \leqslant C h^{1+\gamma} .
$$

If $d_{+} \geqslant d_{-},(5.19)$ holds with $\gamma=2 d_{-}>0$. This is immediate, since $0 \leqslant u_{1}, u_{2} \leqslant 1$. For $d_{+}<d_{-}$, the left-hand side of (5.19) is monotone in $\tau$ 
and therefore does not exceed

$$
\begin{aligned}
& 2 h^{2 d_{+}-1} \int_{0}^{1} v_{1}^{d_{+}-d_{-}} \mathrm{d} v_{1} \int_{0}^{v_{1}}\left(v_{1}-v_{2}\right)^{2 d_{-}-1} v_{2}^{d_{+}-d_{-}} \mathrm{d} v_{2} \\
& =2 h^{2 d_{+}-1} \int_{0}^{1} v_{1}^{2 d_{+}} \mathrm{d} v_{1} \int_{0}^{1}(1-w)^{2 d_{-}-1} w^{d_{+}-d_{-}} \mathrm{d} w=O\left(h^{2 d_{+}-1}\right),
\end{aligned}
$$

which yields again (5.19) with $\gamma=2 d_{+}>0$. This concludes the proof of the theorem.

Theorem 5.2. Let $X_{t}:=B(\mathrm{~d}) G \varepsilon_{t}$. Assume that the assumptions of Theorem 5.1 are satisfied. Then

$$
\begin{aligned}
& N^{-d_{+}-1 / 2} \sum_{t=1}^{[N \tau]} X_{t} \longrightarrow_{D[0,1]} \frac{\bar{g}}{\Gamma\left(d_{+}\right)} W_{d_{+}}^{+}(\tau) \quad \text { if } \quad d_{+}>d_{-}, \\
& N^{-d_{--1}-2} \sum_{t=1}^{[N \tau]} X_{t} \longrightarrow_{D[0,1]} \frac{\bar{g} d_{+}}{d_{-} \Gamma\left(d_{-}\right)} V_{d_{+}, d_{-}}(\tau) \quad \text { if } \quad d_{+}<d_{-},
\end{aligned}
$$

where $V_{d_{+}, d_{-}}(\tau)$ is defined in (4.4).

$\mathrm{P} \mathrm{r}$ o o f. It is similar to the proof of Theorem 5.1, so we just sketch it. Let $J_{N}, J$ stand for the left- and the right-hand sides of (5.20), respectively, when $\tau=1$. Then $J_{N}=\int h_{N} \mathrm{~d} Z_{N}, J=\int h \mathrm{~d} Z$, where $Z_{N}, Z$ are the same as in the proof of Theorem 5.1, and where $h_{N}=h_{N}(x), h=h(x), x \in \mathbf{R}$, are defined by

$$
h_{N}(x):=N^{-d_{+}} \begin{cases}\sum_{t=1}^{N}(b \star g)_{t-s}(t), & x \in\left(\frac{s-1}{N}, \frac{s}{N}\right], \quad x \in(-\infty, 1], \\ 0, & \text { otherwise }\end{cases}
$$

and

$$
h(x):=\frac{\bar{g}}{\Gamma\left(d_{+}\right)} \begin{cases}\int_{0}^{1}(t-x)^{d_{+}-1} \mathrm{~d} t, & x \in[0,1], \\ 0, & \text { otherwise. }\end{cases}
$$

Then, the convergence $J_{N} \rightarrow$ law $J$ follows from

$$
\left\|h_{N}-h\right\|_{2} \rightarrow 0
$$

First, using Lemma $5.1,(5.4)$ and (5.6), it is easy to establish that $\left\|\left(h_{N}-h\right) \mathbf{1}_{[0,1]}\right\|_{2} \rightarrow 0$. Then it remains to prove

$$
\left\|h_{N} \mathbf{1}_{(-\infty, 0)}\right\|_{2} \rightarrow 0
$$

Now, by definition of $h_{N}$ and using Lemma 5.1, (5.4), (5.6), (5.18), we obtain

$$
\left\|h_{N} \mathbf{1}_{(-\infty, 0)}\right\|_{2}^{2} \leqslant C N^{-1-2 d_{+}} \sum_{s<0} \sum_{t_{1}, t_{2}=1}^{N}\left|(b \star g)_{t_{1}-s}\left(t_{1}\right)(b \star g)_{t_{2}-s}\left(t_{2}\right)\right|
$$




$$
\begin{aligned}
\leqslant & C N^{-1-2 d_{+}} \sum_{s<0} \sum_{t_{1}, t_{2}=1}^{N}\left|t_{1}-s\right|^{d_{+}-1}\left|t_{2}-s\right|^{d_{+}-1} \\
& \times\left(|s|^{2\left(d_{-}-d_{+}\right)}+\left(|s|^{-\delta_{1}}+\left|t_{1}\right|^{-\delta_{1}}\right)\left(|s|^{-\delta_{1}}+\left|t_{2}\right|^{-\delta_{1}}\right)\right) \\
= & O\left(N^{2\left(d_{-}-d_{+}\right)}+N^{-2 \delta_{1}}\right)=o(1)
\end{aligned}
$$

in view of $d_{-}<d_{+}$. This proves (5.25) and the one-dimensional convergence in (5.20) for $\tau=1$.

We turn now to the proof of (5.21) for $\tau=1$. We need again to verify (5.24), with

$$
h_{N}(x):=N^{-d_{-}} \begin{cases}\sum_{t=1}^{N}(b \star g)_{t-s}(t), & x \in\left(\frac{s-1}{N}, \frac{s}{N}\right], \quad x \in(-\infty, 1], \\ 0, & \text { otherwise, }\end{cases}
$$

and

$$
h(x):=\frac{\bar{g} d_{+}}{d_{-} \Gamma\left(d_{-}\right)} \begin{cases}(-x)^{d_{-}-d_{+}} \int_{0}^{1}(t-x)_{+}^{d_{+}-1} \mathrm{~d} t, & x \in(-\infty, 0), \\ 0, & \text { otherwise. }\end{cases}
$$

Using $d_{-}>d_{+}$and Lemma 5.1, (5.4), and (5.6), it follows easily that $\left\|h_{N} \mathbf{1}_{[0,1]}\right\|_{2} \rightarrow 0$ and $\left\|\left(h_{N}-h\right) \mathbf{1}_{(-\infty, 0)}\right\|_{2} \rightarrow 0$. The remaining details including the proof of tightness in both convergences (5.20) and (5.21) are similar to the proof of Theorem 5.1. Theorem 5.2 is proved.

\section{Appendix: Proofs of Proposition 4.1 and Lemma 5.1.}

Proof of Proposition 4.1. In the sequel, $C$ denotes a generic constant which may be different at each occurrence.

(i) Clearly, $\int_{0}^{\tau}\left(\int_{s}^{\tau}(t-s)^{d-1} \mathrm{~d} t\right)^{2}<\infty$ for $d \in\left(0, \frac{1}{2}\right)$, implying that the stochastic integral $W_{d}^{+}(\tau)$ is well defined. Next,

$$
\int_{0}^{\infty} \mathrm{d} s\left(\int_{0}^{\tau} t^{d_{+}-d_{-}}(t+s)^{d_{-}-1} \mathrm{~d} t\right)^{2}=I_{1}+I_{2}
$$

where

$$
\begin{aligned}
I_{1} & :=\int_{1}^{\infty} \mathrm{d} s\left(\int_{0}^{\tau} t^{d_{+}-d_{-}}(t+s)^{d_{-}-1} \mathrm{~d} t\right)^{2} \\
& \leqslant \int_{1}^{\infty} s^{2\left(d_{-}-1\right)} \mathrm{d} s\left(\int_{0}^{\tau} t^{d_{+}-d_{-}} \mathrm{d} t\right)^{2}<\infty \\
I_{2} & :=\int_{0}^{1} \mathrm{~d} s\left(\int_{0}^{\tau} t^{d_{+}-d_{-}}(t+s)^{d_{-}-1} \mathrm{~d} t\right)^{2} \leqslant\left(\int_{0}^{\tau} t^{d_{-}-1} \mathrm{~d} t\right)^{2}<\infty .
\end{aligned}
$$

Therefore, $U_{d_{+}, d_{-}}(\tau)$ is well defined. It is also easy to check that $\int_{0}^{\infty} s^{2\left(d_{-}-d_{+}\right)} \mathrm{d} s\left(\int_{0}^{\tau}(t-s)^{d_{+}-1} \mathrm{~d} t\right)^{2}<\infty$, or the fact that $V_{d_{+}, d_{-}}(\tau)$ is well defined. The statement about self-similarity follows from the scaling property 
$(Z(a u), u)=_{\text {fdd }}\left(a^{1 / 2} Z(u), u\right)$ of the white noise and the scaling properties of the kernels of integrals in (4.2)-(4.4). This proves (i). lations

(ii) The statement about $U_{d_{+}, d_{-}}$and $V_{d_{+}, d_{-}}$follows from respective re-

$$
\begin{gathered}
\int_{0}^{\infty} s^{2\left(d_{-}-d_{+}\right)} \mathrm{d} s\left(\int_{T}^{T+\tau}(t+s)^{d_{+}-1} \mathrm{~d} t\right)^{2} \longrightarrow 0 \\
\int_{0}^{\infty} \mathrm{d} s\left(\int_{T}^{T+\tau} t^{d_{+}-d_{-}}(t+s)^{d_{-}-1} \mathrm{~d} t\right)^{2} \longrightarrow 0
\end{gathered}
$$

for each $\tau>0$, as $T \rightarrow \infty$. It suffices to consider $\tau=1$ only. Then the inner integral in (6.1) does not exceed $(T+s)^{d_{+}-1}$ and therefore the left-hand side of $(6.1)$ does not exceed

$$
T^{2 d_{-}-1} \int_{0}^{\infty} s^{2\left(d_{-}-d_{+}\right)}(1+s)^{2 d_{+}-2} \mathrm{~d} s \leqslant C T^{2 d_{--1}}=o(1),
$$

proving (6.1). The proof of (6.2) follows similarly.

To prove the statement about $W_{d}^{+}(\tau)$, write

$$
W_{d}^{+}(\tau)=W_{d+1 / 2}(\tau)-U_{d, d}(\tau),
$$

see (4.5), where the process $U_{d, d}$ has asymptotically vanishing increments as shown above, while $W_{d+1 / 2}$ has stationary increments. Therefore, $W_{d}^{+}$has asymptotically stationary increments

$$
\left(W_{d}^{+}(\tau+T)-W_{d}^{+}(T), \tau \geqslant 0\right) \longrightarrow_{\mathrm{fdd}}\left(W_{d+1 / 2}(\tau), \tau \geqslant 0\right) .
$$

This proves (ii).

(iii) For a Gaussian process of the form $W(\tau)=\int_{0}^{\infty} g(\tau, s) Z(\mathrm{~d} s)$, the almost sure differentiability on $(0, \infty)$ follows if we show that for any $k \geqslant 1$, the partial derivatives $g^{(k)}(\tau, s)=\partial^{k} g(\tau, s) / \partial \tau^{k}$ exist for each $\tau, s>0$ and are square integrable in $s \in(0, \infty)$ and the integrals $\int_{0}^{\infty}\left(g^{(k)}(\tau, s)\right)^{2} \mathrm{~d} s$ are bounded on compact intervals of $(0, \infty)$ (see, e.g., [10]). The process $U_{d_{+}-d_{-}}(\tau)$ admits this form of representation with

$$
g(\tau, s):=\int_{0}^{\tau} t^{d_{+}-d_{-}}(t+s)^{d_{-}-1} \mathrm{~d} t, \quad \tau, s>0 .
$$

We have

$$
g_{\tau}^{(k)}(\tau, s)=\sum_{\ell=0}^{k-1}\left(\begin{array}{c}
k-1 \\
\ell
\end{array}\right) g_{1}^{(\ell)}(\tau) g_{2}^{(k-1-\ell)}(\tau+s)
$$

where

$$
g_{1}(\tau):=\tau^{d_{+}-d_{-}}, \quad g_{2}(\tau):=\tau^{d_{-}-1}
$$

Then

$$
\left|g_{1}^{(\ell)}(\tau)\right| \leqslant C \tau^{d_{+}-d_{-}-\ell} \quad \text { and } \quad\left|g^{(k-1-\ell)}(\tau+s)\right| \leqslant C(\tau+s)^{d_{-}-k+\ell}
$$


implying

$$
\begin{aligned}
\int_{0}^{\infty}\left(g^{(k)}(\tau, s)\right)^{2} \mathrm{~d} s & \leqslant C \sum_{\ell=0}^{k-1} \tau^{2\left(d_{+}-d_{-}-\ell\right)} \int_{0}^{\infty}(\tau+s)^{2\left(d_{-}-k+\ell\right)} \mathrm{d} s \\
& \leqslant C \sum_{\ell=0}^{k-1} \tau^{2\left(d_{+}-d_{-}-\ell\right)} \int_{0}^{\infty}(\tau+s)^{2\left(d_{-}-1\right)} \mathrm{d} s \\
& =C \sum_{\ell=0}^{k-1} \tau^{2\left(d_{+}-\ell\right)-1} \int_{0}^{\infty}(1+s)^{2\left(d_{-}-1\right)} \mathrm{d} s
\end{aligned}
$$

where the last expression is bounded on $\tau \in(\epsilon, \infty)$ for any $\epsilon>0$ fixed. This proves the a.s. differentiability of $U_{d_{+}, d_{-}}$. For the process $V_{d_{+}, d_{-}}$the proof is similar by taking the function $g(\tau, s)=s^{d_{-}-d_{+}} \int_{0}^{\tau}(t+s)^{d_{+}-1} \mathrm{~d} t$. This proves part (iii) and the proposition.

$\mathrm{Pr}$ o of of $\mathrm{L} \mathrm{e} \mathrm{mm}$ a 5.1. Introduce the following notation. For any integers $s<t$, put

$$
\begin{aligned}
\theta_{t, s}^{+}:=\frac{a_{t-s}(t)}{\psi_{t-s}\left(d_{+}\right)}, & \theta_{t, s}^{-}:=\frac{a_{t-s}(t) \psi_{t}\left(d_{-}\right)}{\psi_{v-s}\left(d_{-}\right) \psi_{t}\left(d_{+}\right)}, \\
\vartheta_{t, s}^{+}:=\frac{b_{t-s}(t)}{\psi_{t-s}\left(d_{+}\right)}, & \vartheta_{t, s}^{-}:=\frac{b_{t-s}(t) \psi_{1-s}\left(d_{+}\right) d_{-}}{\psi_{t-s}\left(d_{+}\right) \psi_{1-s}\left(d_{-}\right) d_{t-1}} .
\end{aligned}
$$

To prove Lemma 5.1, we need the following result.

Lemma 6.1. Let $\left(d_{t}, t \in \mathbf{Z}\right)$ be a sequence such that (1.11) and (5.1) hold. Then there exists $\delta_{2}>0$ such that

$$
\begin{aligned}
\sup _{s: 0<s<t}\left|\theta_{t, s}^{+}-1\right|=O\left(t^{-\delta_{2}}\right), & t \rightarrow+\infty, \\
\sup _{s: s \leqslant 0}\left|\theta_{t, s}^{-}-1\right|=O\left(t^{-\delta_{2}}\right), & t \rightarrow+\infty, \\
\sup _{t: s<t}\left|\vartheta_{t, s}^{+}-1\right|=O\left(s^{-\delta_{2}}\right), & s \rightarrow+\infty, \\
\sup _{t: t>0}\left|\vartheta_{t, s}^{-}-1\right|=O\left(|s|^{-\delta_{2}}\right), & s \rightarrow-\infty .
\end{aligned}
$$

$\mathrm{P} \mathrm{r}$ o o f. Let us prove (6.3). By definition,

$$
\theta_{t, s}^{+}=\frac{a_{t-s}(t)}{\psi_{t-s}\left(d_{+}\right)}=\prod_{s \leqslant k<t}\left(1+\frac{d_{k}-d_{+}}{d_{+}+t-k-1}\right) .
$$

Note that for $t$ large enough, $\beta_{t, k}^{+}:=\left(d_{k}-d_{+}\right) /\left(d_{+}+t-k-1\right)$ is arbitrary small uniformly in $k<t$. Indeed, by boundedness of $\left(d_{t}, t \in \mathbf{Z}\right)$, for any $\epsilon>0$ one can find $K<\infty$ such that $\left|\beta_{t, k}^{+}\right|<\epsilon$ for any $t \in \mathbf{Z}, k<t-K$. On the other hand, $\max _{t-K \leqslant k<t}\left|\beta_{t, k}^{+}\right| \rightarrow 0(t \rightarrow+\infty)$, because $\max _{t-K \leqslant k \leqslant t}\left|d_{k}-d_{+}\right| \rightarrow 0$ $(t \rightarrow+\infty)$. Since $\mathrm{e}^{-2|x|} \leqslant 1+x \leqslant \mathrm{e}^{2|x|}$ for $|x|$ small enough, we obtain for $t$ sufficiently large

$$
\exp \left\{-2 \sum_{s \leqslant k<t}\left|\beta_{t, k}^{+}\right|\right\} \leqslant \theta_{t, s}^{+} \leqslant \exp \left\{2 \sum_{s \leqslant k<t}\left|\beta_{t, k}^{+}\right|\right\},
$$


where, by (5.1),

$$
\sum_{s \leqslant k<t}\left|\beta_{t, k}^{+}\right| \leqslant C \sum_{1 \leqslant k<t} k^{-\delta}(t-k)^{-1} .
$$

As $t$ tends to infinity, the right-hand side is dominated by

$$
C \int_{1}^{t-1} x^{-\delta}(t-x)^{-1} \mathrm{~d} x=C t^{-\delta} \int_{1 / t}^{1-1 / t} x^{-\delta}(1-x)^{-1} \mathrm{~d} x \leqslant C\left(t^{-\delta} \ln t+t^{-1}\right) .
$$

This proves (6.3).

Next, let us prove (6.4). We have for $s<0<t$

$$
\theta_{t, s}^{-}=\prod_{s \leqslant p<0}\left(1+\frac{d_{p}-d_{-}}{d_{-}+t-p-1}\right) \prod_{0 \leqslant k<t}\left(1+\frac{d_{k}-d_{+}}{d_{+}+t-k-1}\right) .
$$

As above, for any $s<0$ and some $t>t_{0}>0$ large enough,

$\exp \left\{-2 \sum_{s \leqslant p<0}\left|\beta_{t, p}\right|-2 \sum_{0 \leqslant k<t}\left|\beta_{t, k}^{+}\right|\right\} \leqslant \theta_{t, s}^{-} \leqslant \exp \left\{2 \sum_{s \leqslant p<0}\left|\beta_{t, p}\right|+2 \sum_{0 \leqslant k<t}\left|\beta_{t, k}^{+}\right|\right\}$.

Here, by (5.1),

$$
\begin{aligned}
\sum_{s \leqslant p<0}\left|\beta_{t, p}\right| & \leqslant C \sum_{s \leqslant p<0}|p|^{-\delta}(t-p)^{-1} \leqslant C \sum_{k>0} k^{-\delta}(t+k)^{-1} \\
& \leqslant C \int_{1}^{\infty} x^{-\delta}(t+x)^{-1} \mathrm{~d} x \leqslant C\left(t^{-\delta} \ln t+t^{-1}\right)
\end{aligned}
$$

and

$$
\sum_{0 \leqslant k<t}\left|\beta_{t, k}^{+}\right| \leqslant\left|\beta_{t, 0}^{+}\right|+C \sum_{k=1}^{t-1} k^{-\delta}(t-k)^{-1} \leqslant C\left(t^{-1}+t^{-\delta} \ln t\right),
$$

where the last sum was estimated in the proof of (6.3). This proves (6.4).

Let us prove (6.5). By definition,

$$
\vartheta_{t, s}^{+}=\frac{d_{t-1}}{d_{+}} \prod_{s \leqslant k \leqslant t-2}\left(1+\frac{d_{k}-d_{+}}{d_{+}+k-s+1}\right) .
$$

Note that for $s$ large enough, $\lambda_{s, k}^{+}:=\left(d_{k}-d_{+}\right) /\left(d_{+}+k-s+1\right)$ is arbitrary small uniformly in $k \geqslant s$. Consequently, and since $d_{t-1} / d_{+} \rightarrow 1$ as $t \rightarrow+\infty$, for any $\epsilon>0$ and all $s$ sufficiently large we obtain

$$
(1-\epsilon) \exp \left\{-2 \sum_{s \leqslant k \leqslant t-2}\left|\lambda_{s, k}^{+}\right|\right\} \leqslant \vartheta_{t, s}^{+} \leqslant(1+\epsilon) \exp \left\{2 \sum_{s \leqslant k \leqslant t-2}\left|\lambda_{s, k}^{+}\right|\right\},
$$

where, by (5.1),

$$
\sum_{s \leqslant k \leqslant t-2}\left|\lambda_{s, k}^{+}\right| \leqslant C \sum_{s \leqslant k} k^{-\delta}(k-s+1)^{-1} \leqslant C s^{-\delta},
$$

proving (6.5). 
Finally, let us prove (6.6). We have for $s<0<t$

$$
\begin{aligned}
\vartheta_{t, s}^{-} & =\frac{d_{-} \psi_{-s}\left(d_{+}\right)}{d_{+} \psi_{-s}\left(d_{-}\right)} \prod_{s \leqslant k \leqslant t-2}\left(1+\frac{d_{k}-d_{+}}{d_{+}+k-s+1}\right) \\
& =\prod_{s \leqslant p<-1}\left(1+\frac{d_{p}-d_{-}}{d_{-}+p-s+1}\right) \prod_{-1 \leqslant k<t-2}\left(1+\frac{d_{k}-d_{+}}{d_{+}+k-s+1}\right) .
\end{aligned}
$$

As above, for any $|s|$ large enough, $s<0$, and all $t>0$ we have

$$
\exp \left\{-2 \sum_{p=s}^{-2}\left|\lambda_{s, p}\right|-2 \sum_{k=-1}^{t-2}\left|\lambda_{s, k}^{+}\right|\right\} \leqslant \vartheta_{t, s}^{-} \leqslant \exp \left\{2 \sum_{p=s}^{-2}\left|\lambda_{s, p}\right|+2 \sum_{k=-1}^{t-2}\left|\lambda_{s, k}^{+}\right|\right\} \text {. }
$$

Here, by (5.1),

$$
\sum_{p=s}^{-2}\left|\lambda_{s, p}\right| \leqslant C \sum_{p=s}^{-2}|p|^{-\delta}(p-s+1)^{-1} \leqslant C\left(|s|^{-1}+|s|^{-\delta} \ln |s|\right)
$$

and

$$
\sum_{k=-1}^{t-2}\left|\lambda_{s, k}^{+}\right| \leqslant\left|\lambda_{s,-1}^{+}\right|+\left|\lambda_{s, 0}^{+}\right|+C \sum_{k=1}^{\infty} k^{-\delta}(k+|s|)^{-1} \leqslant C\left(|s|^{-1}+|s|^{-\delta} \ln |s|\right) .
$$

This proves (6.6) and Lemma 6.1 too.

Return to the proof of Lemma 5.1. Denote $\psi_{j}^{ \pm}:=\psi_{j}\left(d_{ \pm}\right), \phi_{j}^{ \pm}:=$ $\Gamma\left(d_{ \pm}\right)^{-1} j_{+}^{d_{ \pm}-1}(j=0,1,2, \ldots)$. We shall use the well-known relation

$$
\left|\psi_{j}^{ \pm}-\phi_{j}^{ \pm}\right| \leqslant C j_{+}^{d_{ \pm}-2} \quad(j \geqslant 0) .
$$

Let us prove (5.3). Let first $0 \leqslant s \leqslant t$. Then $\Theta_{t, s}^{(1)}=(a \star g)_{t-s}(t)-\bar{g} \psi_{t-s}^{+}=$ $\sum_{k=1}^{5} I_{k}(t, s)$, where

$$
\begin{aligned}
I_{1}(t, s) & :=-\psi_{t-s}^{+} \sum_{i \geqslant t-s} g_{i}, \\
I_{2}(t, s) & :=\sum_{0 \leqslant i<t-s} g_{i}\left(\phi_{t-s-i}^{+}-\phi_{t-s}^{+}\right), \\
I_{3}(t, s) & :=\sum_{0 \leqslant i<t-s} g_{i}\left(\psi_{t-s-i}^{+}-\phi_{t-s-i}^{+}\right), \\
I_{4}(t, s) & :=\left(\phi_{t-s}^{+}-\psi_{t-s}^{+}\right) \sum_{0 \leqslant i<t-s} g_{i}, \\
I_{5}(t, s) & :=\sum_{0 \leqslant i<t-s} g_{i} \psi_{t-s-i}^{+}\left(\theta_{t, s+i}^{+}-1\right) .
\end{aligned}
$$

Then it suffices to show that there exists $\delta_{1}>0$ such that

$$
\left|I_{k}(t, s)\right| \leqslant C(t-s)^{d_{+}-1}(t-s)^{-\delta_{1}}, \quad k=1, \ldots, 5 .
$$

For $k=1$ and $k=4,(6.8)$ is immediate by (2.1) and (6.7), with $\delta_{1}=\delta$ and $\delta_{1}=1$, respectively. Next, split $I_{2}(t, s)=\sum_{(t-s) / s<i<t-s}+\sum_{0 \leqslant i \leqslant(t-s) / s}=$ : 
$I_{21}(t, s)+I_{22}(t, s)$. Then

$\left|I_{21}(t, s)\right| \leqslant C(t-s)^{-1-\delta} \sum_{(t-s) / s<i<t-s}\left((t-s-i)^{d_{+}-1}+(t-s)^{d_{+}-1}\right) \leqslant C(t-s)^{d_{+}-1-\delta}$

satisfies (6.8) and, assuming without loss of generality that $\delta<1$,

$$
\begin{aligned}
I_{22}(t, s) & \leqslant C \sum_{0 \leqslant i \leqslant(t-s) / 2} i_{+}^{-1-\delta}\left((t-s-i)^{d_{+}-1}-(t-s)^{d_{+}-1}\right) \\
& \leqslant C \sum_{0 \leqslant i \leqslant(t-s) / 2} i_{+}^{-\delta}(t-s-i)^{d_{+}-2} \leqslant C(t-s)^{d_{+}-1-\delta} .
\end{aligned}
$$

So, (6.8) is proved for $k=2$. For $k=3,(6.8)$ follows by (6.7) in a similar way. Finally, by Lemma $6.1,(6.3)$,

$$
\left|I_{5}(t, s)\right| \leqslant C t^{-\delta_{2}} \sum_{0 \leqslant i<t-s} i_{+}^{-1-\delta}(t-s-i)^{d_{+}-1} \leqslant C(t-s)^{-\delta_{2}}(t-s)^{d_{+}-1} .
$$

Now consider the case $s<0 \leqslant t$. Similarly as above, we shall split $\Theta_{t, s}^{(1)}=(a \star g)_{t-s}(t)-\bar{g} \psi_{t-s}^{-} \psi_{t}^{+} / \psi_{t}^{-}=\sum_{k=1}^{6} I_{k}^{\prime}(t, s)$ and show, in order to prove (5.5), that there exist $\delta_{3}>0$ and $\delta_{4}>0$ such that

$$
\left|I_{k}^{\prime}(t, s)\right| \leqslant C(t-s)^{d_{-}-1} t^{d_{+}-d_{-}}\left(t^{-\delta_{3}}+(-s)^{-\delta_{4}}\right), \quad k=1, \ldots, 6 .
$$

Here

$$
\begin{aligned}
& I_{1}^{\prime}(t, s):=-\psi_{t-s}^{-} \frac{\psi_{t}^{+}}{\psi_{t}^{-}} \sum_{i \geqslant-s} g_{i}, \\
& I_{2}^{\prime}(t, s):=\frac{\psi_{t}^{+}}{\psi_{t}^{-}} \sum_{0 \leqslant i<-s} g_{i}\left(\phi_{t-s-i}^{-}-\phi_{t-s}^{-}\right), \\
& I_{3}^{\prime}(t, s):=\frac{\psi_{t}^{+}}{\psi_{t}^{-}} \sum_{0 \leqslant i<-s} g_{i}\left(\psi_{t-s-i}^{-}-\phi_{t-s-i}^{-}\right), \\
& I_{4}^{\prime}(t, s):=\left(\phi_{t-s}^{-}-\psi_{t-s}^{-}\right) \frac{\psi_{t}^{+}}{\psi_{t}^{-}} \sum_{0 \leqslant i<-s} g_{i}, \\
& I_{5}^{\prime}(t, s):=\frac{\psi_{t}^{+}}{\psi_{t}^{-}} \sum_{0 \leqslant i<-s} g_{i} \psi_{t-s-i}^{-}\left(\theta_{t, s+i}^{-}-1\right), \\
& I_{6}^{\prime}(t, s):=\sum_{-s \leqslant i \leqslant t-s} g_{i} a_{t-s-i}(t) .
\end{aligned}
$$

Let us first estimate the last term. By Lemma 6.1, (6.3), we have $\left|I_{6}^{\prime}(t, s)\right| \leqslant$ $C \sum_{|s| \leqslant i \leqslant t+|s|} i^{-1-\delta}(t+|s|-i)^{d_{+}-1}$. There are two cases: $|s| \geqslant t$ and $|s|<t$. For $|s| \geqslant t$,

$$
\begin{aligned}
\left|I_{6}^{\prime}(t, s)\right| & \leqslant C(t+|s|)^{-1-\delta} \sum_{|s| \leqslant i<t+|s|}(t+|s|-i)^{d_{+}-1} \\
& \leqslant C(t+|s|)^{-1-\delta} t^{d_{+}} \leqslant C t^{d_{+}-d_{-}}(t+|s|)^{d_{-}-1}(t+|s|)^{-\delta} .
\end{aligned}
$$


For $|s|<t$, by splitting the $\operatorname{sum} \sum_{|s| \leqslant i<t+|s|}=\sum_{|s| \leqslant i<(t+|s|) / 2}+\sum_{(t+|s|) / 2 \leqslant i \leqslant t+|s|}$, we obtain

$\left|I_{6}^{\prime}(t, s)\right| \leqslant C(t+|s|)^{d_{+}-1}|s|^{-\delta}+C(t+|s|)^{d_{+}-1-\delta} \leqslant C t^{d_{+}-d_{-}}(t+|s|)^{d_{-}-1}|s|^{-\delta}$, thus proving (6.9) for $k=6$.

Next, by Lemma 6.1, (6.4), we have

$$
\left|I_{5}^{\prime}(t, s)\right| \leqslant C t^{d_{+}-d_{-}} \sum_{0 \leqslant i<t+|s|} i_{+}^{-1-\delta}(t+|s|-i)^{d_{-}-1} t^{-\delta_{2}} \leqslant C t^{d_{+}-d_{-}}(t+|s|)^{d_{-}-1} t^{-\delta_{2}},
$$

proving (6.9) for $k=5$. In the case $k=4$, use (6.7) to obtain $\left|I_{4}^{\prime}(t, s)\right| \leqslant$ $C(t+|s|)^{d_{--2}} t^{d_{+}-d_{-}} \leqslant C t^{d_{+}-d_{-}}(t+|s|)^{d_{-}-1} t^{-1}$, and, similarly,

$$
\left|I_{3}^{\prime}(t, s)\right| \leqslant C t^{d_{+}-d_{-}} \sum_{0 \leqslant i<t+|s|} i_{+}^{-1-\delta}(t+|s|-i)^{d_{-}-2} \leqslant C t^{d_{+}-d_{-}}(t+|s|)^{d_{-}-1-\delta} .
$$

For $k=1,2,(6.9)$ follows analogously. This proves (5.3) and (5.5).

Let us prove (5.4) and (5.6). For $0 \leqslant s \leqslant t$ the proof is similar by Lemma 6.1, (6.5), and is omitted. Consider the case $s<0 \leqslant t$. Split $\Theta_{t, s}^{(2)}=(b \star g)_{t-s}(t)-\bar{g} \psi_{t-s}^{+}\left(d_{+} \psi_{1-s}^{-} / d_{-} \psi_{1-s}^{+}\right)=\sum_{k=1}^{4} J_{k}(t, s)$, where

$$
\begin{aligned}
J_{1}(t, s) & :=-\psi_{t-s}^{+} \frac{d_{+} \psi_{1-s}^{-}}{d_{-} \psi_{1-s}^{+}} \sum_{i \geqslant-s} g_{i}, \\
J_{2}(t, s) & :=\frac{d_{+}}{d_{-}} \sum_{0 \leqslant i<-s} g_{i}\left(\psi_{t-s-i}^{+} \frac{\psi_{1-s-i}^{-}}{\psi_{1-s-i}^{+}}-\psi_{t-s}^{+} \frac{\psi_{1-s}^{-}}{\psi_{1-s}^{+}}\right), \\
J_{3}(t, s) & :=\sum_{0 \leqslant i<-s} g_{i}\left(b_{t-s-i}(t)-\psi_{t-s-i}^{+} \frac{\psi_{1-s-i}^{-}}{\psi_{1-s-i}^{+}}\right), \\
J_{4}(t, s) & :=\sum_{-s \leqslant i \leqslant t-s} g_{i} b_{t-s-i}(t) .
\end{aligned}
$$

Using (1.11), (2.1), (6.7), and Lemma 6.1, (6.6), the proof of the bound (5.6), with factor $|s|^{\left(d_{-}-d_{+}\right) \vee 0}$ replaced by $|s|^{d_{-}-d_{+}}$, for terms $J_{k}(t, s), k=1,2,3$, is similar to the estimation of the corresponding terms in the decomposition $\Theta_{t, s}^{(1)}=\sum_{k=1}^{6} I_{k}^{\prime}(t, s)$ above, and is omitted. Consider the last term $J_{4}(t, s)$. Here $0 \leqslant t-s-i$ and we use the bound $\left|b_{t-s-i}(t)\right| \leqslant C \psi_{t-s-i}^{+}$which follows from Lemma 6.1, (6.5). Together with (2.1), it implies

$$
\left|J_{k}(t, s)\right| \leqslant C \sum_{i=|s|}^{t+|s|} i^{-1-\delta}(t+|s|-i)^{d_{+}-1}=\Sigma_{1}+\Sigma_{2},
$$

where $\Sigma_{1}:=\sum_{|s| \leqslant i<(t+|s|) / 2}, \Sigma_{2}:=\sum_{(t+|s|) / 2 \leqslant i<t+|s|}$. Then we obtain

$$
\begin{aligned}
& \Sigma_{1} \leqslant C(t+|s|)^{d_{+}-1} \sum_{i \geqslant|s|} i^{-1-\delta} \leqslant C(t-s)^{d_{+}-1}|s|^{-\delta}, \\
& \Sigma_{2} \leqslant C(t+|s|)^{-1-\delta} \sum_{i=1}^{(t+|s|) / 2} i^{d_{+}-1} \leqslant C(t-s)^{d_{+}-1-\delta},
\end{aligned}
$$

thus proving the bound (5.6) for $J_{4}(t, s)$ and Lemma 5.1 too. 


\section{REFERENCES}

1. Benassi A., Cohen S., Istas J. Identifying the multifractional function of a Gaussian process. - Statist. Probab. Lett., 1998, v. 39, № 4, p. 337-345.

2. Биллингсли П. Сходимость вероятностных мер. М.: Наука, 1977, 351 с.

3. Brockwell P. J., Davis R. A. Time Series: Theory and Methods. New York: SpringerVerlag, 1991, $577 \mathrm{p}$.

4. Brużaitè K., Surgailis D., Vaičiulis $M$. Time-varying fractionally integrated processes with finite or infinite variance and nonstationary long memory. - Acta Appl. Math., 2007, v. 96, № 1-3, p. 99-118.

5. Cox D.R. Long-range dependence: A review. Statistics: An Appraisal. Ed. by H. A. David and H. T. David. Iowa State Univ. Press, Iowa, 1984, p. 55-74.

6. Давыдов Ю. А. Принцип инвариантности для стационарных процессов. - Теория вероятн. и ее примен., 1970 , т. 15 , в. 3 , с. $498-509$.

7. Dehling H., Philipp W. Empirical process techniques for dependent data. - Empirical Process Techniques for Dependent Data. Ed. by H. Dehling, T. Mikosch, M. Sørensen. Boston: Birkhäuser, p. 3-113.

8. Doukhan P., Oppenheim G., Taqqu M.S. (eds.). Theory and Applications of LongRange Dependence. Boston: Birkhäuser, 2003, 716 p.

9. Doukhan P., Lang G., Surgailis D. Randomly fractionally integrated processes. Liet. Mat. Rink., 2007, v. 47, № 1, p. 3-28.

10. Кутояни Ю. А. Разложение оценки максимального правдоподобия по степеням диффузии. - Теория вероятн. и ее примен., 1984, т. 29, в. 3, с. 452-463.

11. Lamperti J. Semi-stable stochastic processes. - Trans. Amer. Math. Soc., 1962, v. 104 , p. $62-78$.

12. Lang G., Soulier $P$. Convergence de mesures spectrales aléatoires et applications à des principes d'invariance. - Stat. Inference Stoch. Process, 2000, v. 3, № 1-2, p. 41-51.

13. Marinucci D., Robinson P. M. Alternative forms of fractional Brownian motion. J. Statist. Plann. Inference, 1999, v. 80, № 1-2, p. 111-122.

14. Peltier R., Lévy Véhel J. Multifractional Brownian Motion. Technical report № 2645. INRIA, 1995.

15. Philippe A., Surgailis D., Viano M.-C. Time-varying fractionally integrated processes with nonstationary long memory. - Pub. IRMA Lille, 2004, v. 61, № 9 .

16. Philippe A., Surgailis D., Viano M.-C. Invariance principle for a class of nonstationary with long memory processes. - C. R. Math. Acad. Sci. Paris, 2006, v. 342, № 4, p. 269-274.

17. Surgailis $D$. Non-CLTs: $U$-statistics, multinomial formula and approximations of multiple Itô-Wiener integrals. - Theory and Applications of Long-Range Dependence. Ed. by P. Doukhan, G. Oppenheim, and M.S. Taqqu. Boston: Birkhäuser, 2003, p. $120-142$.

18. Surgailis $D$. Stable limits of sums of bounded functions of long memory moving averages with finite variance. - Bernoulli, 2004, v. 10, № 2, p. 327-355.

19. Surgailis $D$. Nonhomogeneous fractional integration and multifractional processes. Stochastic Process. Appl., 2007 (to appear).

Поступила в редакцию

5.X.2005 